\title{
Microalgae: Coming to a Food Near You
}

\section{Patricia Lopez-Sanchez studies the flow properties of microalgae, with the aim of incorporating this nutritional powerhouse into new food products.}

\author{
By Katherine Wright
}

$\square$

atricia Lopez-Sanchez did not set out to be an academic. After completing an undergraduate degree in chemistry in Spain, Lopez-Sanchez moved to the Netherlands to work for Unilever, a company that makes products ranging from ice cream to shampoo. During her eight years there, she got a taste for academic life by doing an industrial Ph.D. and decided to move to Australia for a postdoc. She now lives in Sweden, where she has worked at a startup company developing a drink for marathon runners and at the Research Institutes of Sweden helping companies refine recipes for food products. Today she works at Chalmers University of Technology, Sweden, where she studies the structure and flow properties of microalgae that companies want to incorporate into snacks and drinks. Physics spoke to Lopez-Sanchez about her fascination for these trending plants, what they taste like in foods, and her

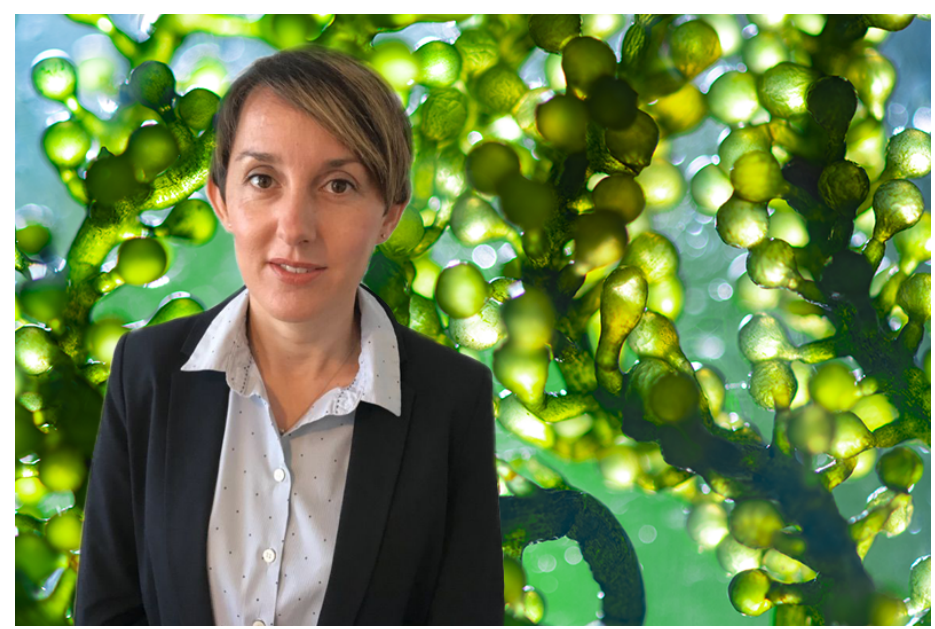

Credit: P. Lopez-Sanchez; APS/Carin Cain; chokniti/stock.adobe.com cross-disciplinary, zig-zagging career.

All interviews are edited for brevity and clarity.

Food science is a multidisciplinary field. What's it like working with people from such different backgrounds? I find it very rewarding, and it's one of the things I like most about my job. I collaborate with chemists, sensory scientists, physicists, and plant biologists. Then there are also the non-scientists who try out the products I've helped develop. It's really a collaborative effort-you can't do work on foods and stay in your silo.

\section{What is it about algae that captures your attention?} Algae have a unique nutritional profile. Most land plants are rich in carbohydrates, some are rich in proteins, but many algae are rich in both. Algae also have interesting lipid profiles, as they contain all the omega fatty acids found in fish. These fats-and the flavors they contain-make algae particularly attractive as plant-based alternatives for fish.

\section{Other than their nutritional profile, are there other} advantages to making food from algae?

Algae grow much faster than land plants, and they need a lot less square footage to cultivate. That means we could grow huge volumes of algae on a small footprint of the sea or the land. (While large algae are grown in the sea, microalgae are mainly grown on land.)

\section{What properties of algae do you study?}

I look at their microstructure and their rheology, which describes how suspensions of the algae flow when subjected to 
a force. These properties determine the texture of a food product, which influences both the eating and digesting experiences. Rheology and structure affect how a food feels in your mouth, how easy it is to swallow, and how the food disintegrates and releases its nutrients in the stomach and in the intestine.

The rheology and structure of a food are also important for manufacturing, as they play a role in how a food is processed. When setting up a manufacturing unit, a company needs to know if the food will be runny like milk or gloopy like mayonnaise, so that they can determine what machinery they will need to get the food into packaging.

\section{What have you learned about the rheology and the structure of the algae that you study?}

I have looked at three species of microalgae-Nannochloropsis gaditana, Scenedesmus almeriensis, and Spirulina platensis. These species have a similar protein content but a different carbohydrate composition, leading to distinct microstructures and rheological behaviors, something my colleagues and I have attributed to the properties of their cell walls.

Spirulina, for example, has soft cell walls that are easy to break via a mechanical or thermal treatment. Under the conditions that we've tested, the rheological properties of the alga resemble those of a gel. Nannochloropsis, on the other hand, has harder cell walls and behaves like a suspension of particles. These different properties mean that we would use the two algae to make different foods.

\section{What does it take to go from a study like yours that}

\section{looks at a fundamental property of a plant to making a food product?}

Time. It takes a lot of iterations of a product before it is given to a panel to test. That is one of the reasons this kind of analysis is done. It's much cheaper to refine a product's properties in the lab than through multiple tasting sessions, each of which requires a panel of specially trained people.

\section{Have you tried any foods made from the algae that you've studied?}

Yes. A snack and a drink. The snack resembled a green cheese doodle, a popular puff snack in Sweden. The concentration of the microalgae in the snack was very low, and even though it didn't have any salt or added flavor, it tasted ok. The drink, on the other hand, tasted like the sea and definitely needed to have some other ingredients added to improve its flavor.

\section{Finally, what made you move from industry to academia?}

Research projects have a different life cycle in industry than in academia. In a company, the project stops as soon as you find a formulation for a product that works. But for me, finding that formulation is where everything starts to get interesting: Once I have a formulation that works, I want to know why it works, why others didn't work, and whether I can predict if some other recipe will be effective. Answering those questions is the fun part.

Katherine Wright is the Deputy Editor of Physics. 